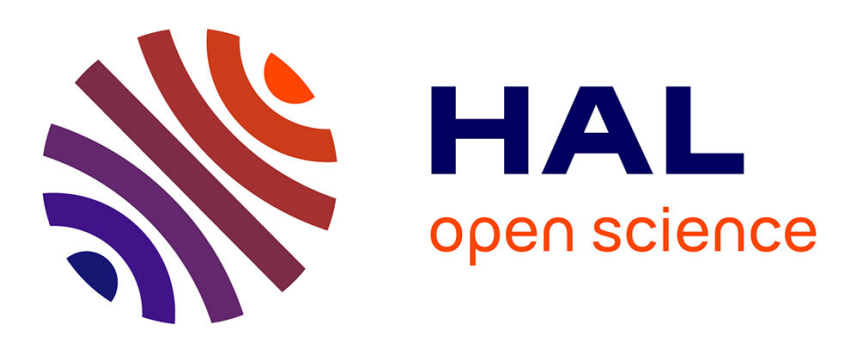

\title{
On the structure and composition of nanoscale TiAlN/VN multilayers
}

Zhaoxia Zhou, Mark Rainforth, Uwe Falke, Meiken Falke, Andrew Bleloch, Papken Hovsepian

\section{- To cite this version:}

Zhaoxia Zhou, Mark Rainforth, Uwe Falke, Meiken Falke, Andrew Bleloch, et al.. On the structure and composition of nanoscale TiAlN/VN multilayers. Philosophical Magazine, 2007, 87 (06), pp.967-978. 10.1080/14786430601019433 . hal-00513784

\section{HAL Id: hal-00513784 \\ https://hal.science/hal-00513784}

Submitted on 1 Sep 2010

HAL is a multi-disciplinary open access archive for the deposit and dissemination of scientific research documents, whether they are published or not. The documents may come from teaching and research institutions in France or abroad, or from public or private research centers.
L'archive ouverte pluridisciplinaire HAL, est destinée au dépôt et à la diffusion de documents scientifiques de niveau recherche, publiés ou non, émanant des établissements d'enseignement et de recherche français ou étrangers, des laboratoires publics ou privés. 


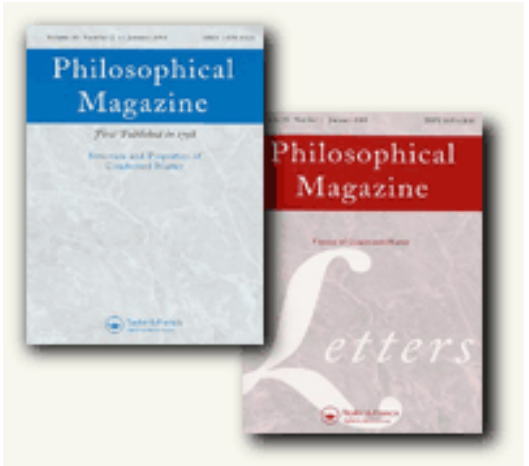

\section{On the structure and composition of nanoscale TiAIN/VN multilayers}

\begin{tabular}{|c|c|}
\hline Journal: & Philosophical Magazine \& Philosophical Magazine Letters \\
\hline Manuscript ID: & TPHM-04-Sep-0024.R2 \\
\hline Journal Selection: & Philosophical Magazine \\
\hline $\begin{array}{r}\text { Date Submitted by the } \\
\text { Author: }\end{array}$ & 12-Sep-2006 \\
\hline Complete List of Authors: & $\begin{array}{l}\text { Zhou, Zhaoxia; The University of Sheffield, Department of } \\
\text { Engineering Materials } \\
\text { Rainforth, Mark; The University of Sheffield, Department of } \\
\text { Engineering Materials } \\
\text { Falke, Uwe; SuperSTEM, Daresbury Laboratory } \\
\text { Falke, Meiken; SuperSTEM, Daresbury Laboratory } \\
\text { Bleloch, Andrew; SuperSTEM, Daresbury Laboratory } \\
\text { Hovsepian, Papken; Sheffield Hallam University, Materials and } \\
\text { Engineering Research Institute }\end{array}$ \\
\hline Keywords: & EELS, aberration correction \\
\hline Keywords (user supplied): & TiAIN/VN multilayers, STEM-HAADF \\
\hline
\end{tabular}

\section{S) ScholaroNE \\ Manuscript Central}


The chemical and physical structure of a TiAlN/VN multilayer, of average layer thickness $3.4 \pm 0.4 \mathrm{~nm}$, was characterised using a spherical aberration corrected STEM, utilising a nominal $0.1 \mathrm{~nm}$ beam, by HAADF and EELS. The interface between layers was shown to be rough, with local thickness variations evident in layer thickness. Chemical mixing between layers was identified, consistent with numerical modelling of the deposition flux and layer growth. The implications of the compositional modulation are discussed.

Keywords: TiAlN/VN multilayers; STEM-HAADF; EELS; spherical aberration correction

\section{Introduction}

Nanoscale multilayers coatings, such as TiAlN/CrN and TiAlN/VN are becoming increasingly important for wear resistant applications, a result of their excellent hardness, resistance to oxidation (and hence their potential in unlubricated milling) and low, stable, friction coefficients under dry contact conditions. Multilayer structures possess novel properties once the multilayer wavelength is in the $\mathrm{nm}$ range (see, for example, the recent Scripta Mater. View point set No. 34 [1]), where the rule of mixtures of the two components no longer applies, and the multilayer exhibits substantially greater hardness than monolithic coatings of the individual constituents. There are several postulated models for the origin of exceptional hardness in multilayers, for example, proposed mechanisms include dislocation hindrance at the layer interfaces, where the stress required to propagate a dislocation through the interface is proportional to the differences in shear moduli between the two layers (Koehler force) [2,3,4] and coherency strains from lattice mismatch of the two materials in each layer of the bi-layers [5]. Although the hardness of the multilayer is a function of the layer thickness (e.g. being a maximum at around 3-4nm for TiAlN/CrN [6]), the optimum thickness cannot be predicted by extrapolation of the Hall-Petch relationship [5,7,8], which highlights the importance of the interfacial structure and residual stress-state.

Nitride multilayers such as TiAlN/VN are isostructural and mutually miscible. Thus, mixing of the constituents is likely during deposition leading to a broadening of the interface. 
While it is still not clear whether dislocation motion is a determinant of hardness in nitride coatings, it is nevertheless clear that the degree of this interfacial mixing will have a strong effect on mechanical properties. The degree of mixing is a function of the deposition conditions. Multilayers are usually deposited using magnetron sputtering. Where a fixed substrate with a movable shutter is used, the degree of mixing between layers is minimised. However, in industrial practice the work-piece is usually rotated between 4 targets, with 3fold rotation of the specimen essential to provide homogenous deposition on 3-dimensional shaped surfaces, such as cutting tools. This promotes greater mixing of the constituents than for a shutter system.

There is currently no clear understanding of the degree of mixing between layers for nitride multilayers and therefore the structure of the interface for multilayer coatings produced under such conditions. The effect of 3-fold rotation on the film growth rate has been modelled for unbalanced magnetron deposition, which identified a secondary modulation superimposed on the basic periodicity in as-deposited superlattice coatings, a result of the substrate rotation $[6,9,10,11,12,13]$. Validation of the oscillation structure has been attempted by glow discharge optical emission spectroscopy (GDOES) and Auger electron spectroscopy (AES) depth profiling [9], but these failed to reveal the details of high frequency oscillations. Recently Zhou et al. [13], used energy filtered TEM, with a resolution around the $1 \mathrm{~nm}$ level, to characterise the elemental distribution between layers. This work was largely consistent with the numerical models of Rother et al. $[9,10,11,12,13]$, but the $1 \mathrm{~nm}$ spatial resolution was insufficient to determine the true elemental distribution mainly due to spherical and chromatic aberrations of the round electromagnetic lenses $[14,15]$.

The current work has used a spherical aberration $\left(\mathrm{C}_{\mathrm{s}}\right)$ corrected scanning transmission electron microscopy (STEM) with $0.1 \mathrm{~nm}$ electron probe to determine chemical distribution through individual layers in a TiAlN/VN multilayer coating, and the experimental results are compared with the numerical model of Zhou et al. [13].

\section{Experimental}

The full details of the deposition conditions are given elsewhere [6]. TiAlN/VN multilayer coatings were grown on $\mathrm{Si}(100)$ single crystal substrates in an industrial scale physical vapour deposition coating machine (Hauzer HTC 1000-4). Deposition was undertaken using the Arc Bond Sputter (ABS) process by reactive unbalanced magnetron sputtering, in a $\mathrm{Ar}+\mathrm{N}_{2}$ atmosphere (a partial pressure ratio of $\mathrm{pAr}$ : $\mathrm{pN}_{2}=2: 1$ ), with two TiAl 
targets adjacent to two $\mathrm{V}$ targets, without mechanical shielding/shutter or gas flow guide plates. A bias voltage of $-85 \mathrm{~V}$ yielded a mean deposition rate of $\sim 20 \mathrm{~nm} / \mathrm{min}$ with a substrate temperature of $450^{\circ} \mathrm{C}$. The substrate rotation unit provided 3 -fold rotation with the main turntable rotating at a speed of $8 \mathrm{rpm}$ (anti-clockwise) with 12 satellites centred on a circle $\mathrm{D}_{\text {Rot1 }}=\emptyset 500 \mathrm{~mm}$ in diameter. Spindles were mounted on a $2^{\text {nd }}$ fold rotation mechanism, which rotates at a rate determined by a gear ratio of $99 / 27$ to the main rotation, anticlockwise. 10 substrate holders were located on each spindle, on a circle $D_{\text {Rot } 2}=\varnothing 95 \mathrm{~mm}$ in diameter, which were rotated to give 3 -fold rotation $\left(D_{\text {Rot3 }}=\emptyset 5 \mathrm{~mm}\right)$.

TEM cross-sections were prepared in the normal manner: 2 slices of coatings were glued by epoxy with coating face to face, ground and polished to $\sim 50 \mu \mathrm{m}$ and attached to $\mathrm{Cu}$ support ring, followed by argon ion beam milling on a Gatan precision ion polishing system (PIPS) at $\pm 3^{\circ}$. Conventional high resolution TEM (HREM) was performed on a JEOL 2010F UHR (point resolution $\sim 0.19 \mathrm{~nm}$ ) TEM with a field emission gun at $200 \mathrm{kV}$. The specimen was subsequently examined at the SuperSTEM Laboratory in Daresbury, UK. The employed microscope is a dedicated STEM, based on a VG HB501, with a Nion Mark II $\mathrm{C}_{\mathrm{s}}$ corrector, which is capable of sub angstrom ( $\AA$ ) high angle annular dark field (HAADF) resolution. HAADF images were acquired using a $0.1 \mathrm{~nm}$ probe at the start of the experiment (although drift in the system may have led to a small increase in probe size with time), which yielded atomic resolution. The HAADF detector acceptance angle was $(70$ - 210) mrad. The definition of the probe size is mainly based on the spatial frequencies in the HAADF signal which are distinct from noise. However, the lateral intensity profile of the STEM probe, apart from a narrow intense centre, has a relatively broad tail. The FWHM is not very suited to describe such peak shapes. A better parameter is the diameter of the area which contains $50 \%$ of the probe intensity. This can be estimated to be approximately $0.16 \mathrm{~nm}$ for the present measurements. Furthermore, the spatial resolution of the measurements is influenced by probe spreading in the sample. Instabilities of probe position with respect to the beam come into play for longer measurements at one and the same sample position as it is the case with EELS. The spatial delocalisation of the EEL signal at $500 \mathrm{eV}$ energy loss is less than $0.2 \mathrm{~nm}$ [16]. EELS spectra were acquired using an Isaacson/ Scheinfein/ Wardell EELS prism spectrometer, with the same probe size and with the detection angle roughly equal to the beam convergence angle (half angle $24 \mathrm{mrad}$ ). The probe was moved 120 points along a line of $16 \mathrm{~nm}$ (internally calibrated with the Si substrate) and held at each point with an exposure of 4 seconds to acquire the spectrum plus a fast letterbox-like HAADF scan of the area 
around. The effective scan trace was deduced from HAADF images recorded immediately before and after the EEL measurement. The additional letterbox scans were used to identify possible deviations from the assumed constant drift rate. Intensity profiles were then derived by net edge integration of the $\mathrm{N} \mathrm{K}, \mathrm{Ti}_{2,3}$ and $\mathrm{V} \mathrm{L}_{2,3}$ ionisation edges, using the energy widths given in Table 1, following background subtraction, with the background profile determined across the energy range also given in Table 1. In order to obtain relative concentrations, the net edge intensities were normalized by inelastic Hartree-Slater cross sections and it was assumed that the stoichiometry in the investigated area is described by $\mathrm{V}_{1-x} \mathrm{Ti}_{0.5 x} \mathrm{Al}_{0.5 x} \mathrm{~N}$.

\section{Results and Discussion}

Fig. 1 gives a conventional TEM image of the Si substrate, the base layer of VN, which was approximately 200nm thick, and the TiAlN/VN multilayer. The coating exhibited a columnar grain structure, as expected from the deposition conditions. The approximate position and thickness of the individual layers can be seen in Fig. 1 by Fresnel diffraction contrast.

It was found by XRD that $\{111\}$ planes were preferentially aligned parallel to the coating surface, while the residual stress in the coating was $7.9 \mathrm{GPa}$, compressive. Details of the method can be found in Lewis et al. [17] and Luo et al. [18]. Texture was also examined by TEM and selected area diffraction pattern (SADP) study through the thickness of the coating. This indicated a dominance of $\{200\}$ parallel to the coating plane close to the base layer, while $\{111\}$ texture was observed in the outer layers (which maximises the wear resistance of these coatings [19]), consistent with the XRD results. Fig.1 shows the TEM microstructure and SADP (inset) of the bottom of coating.

Fig. 2a shows a conventional HREM image of the multilayer, which fails to reveal layer position and thickness since the TiAlN and VN are isostructural and have similar lattice constants $(0.418 \mathrm{~nm}$ for TiAlN and $0.414 \mathrm{~nm}$ for $\mathrm{VN}$ ) (in this image, the layers run horizontally). However, the image does confirm the coherency/partial coherency between layers, as expected. The (111) planes are aligned approximately parallel with the layers, consistent with the XRD data.

Fig. $2 b$ shows a HAADF image from the same sample, but closer to the base layer than Fig. 2a. The electrons contributing to the HAADF signal are scattered to high angles essentially by Rutherford-type scattering [20]. The signal is sensitive to the atomic number 
since heavier atoms are more likely to scatter to higher angles. The signal of a column of atoms is proportional to the average of the squared atomic numbers of the column, i.e. $\mathrm{Z}^{2}$, if one neglects dynamical effects of electron scattering. As TiAlN and VN are isostructural and intermixing of TiAl and $\mathrm{V}$ is inevitable in the coating, it may be assumed that all columns consist of mixed $\mathrm{Ti}, \mathrm{Al}$ and $\mathrm{V}$. The contrast arises as $\mathrm{V}$-dominant columns have a higher average of the squared atomic number providing a larger HAADF signal than TiAl dominant ones. The $\mathrm{N}$-columns are not visible by this technique due to their low scattering amplitude. The problem of Fresnel diffraction contrast is minimised by using STEM and HAADF because of the loss of transverse coherence in electrons scattered to high angles [21] and detected over a very large angular range. The image again confirms the partial coherency between layers, but also suggests that the interface between individual layers is irregular, even over distances of a few unit cells. Moreover, the HAADF data suggest that in some areas the VN layers may not be fully separated. Within this region, the (200) planes are perpendicular to the layers and therefore the interface between layers is approximately along (220). Thus, this region represents an area where there is a texture mid way between the $\{200\}$ texture near the substrate and the $\{111\}$ outer texture.

Fig. 3 gives further HAADF images close to the base layer (including the base layer itself) and close to the outer region of the coating. The black and white lines in the images indicate the track path along which EEL spectra series were measured. Sample drift was found to be less than $0.4 \mathrm{~nm}$ during an EELS acquisition series by comparing the scanned HAADF images recorded before and after EELS acquisitions. Figs. 3c,d give corresponding EELS integrated net intensities along the line scans. The EEL data indicate that there is mixing between layers, i.e. that there is $\mathrm{Ti}$ present throughout the $\mathrm{VN}$ layer, while $\mathrm{V}$ is present throughout the TiAlN layer.

The assumption regarding the stoichiometry could not be proved since that would require the measurement of loss edge intensities of all four contained elements at the same time. This is not possible in the used microscope. Due to the special electron optics the EELS spectrum is in focus only in a small energy loss range which would not include any of the $\mathrm{Al}$ edges together with the observed ones. The stoichiometry assumption might be wrong for the first few layers of the stack after the VN base layer as in the net edge intensity curves (Fig.3c) the maxima of the Ti signal seem to be shifted against the $\mathrm{V}$ signal minima. This shift could either be caused by a drop of the total signal intensity in that interval or by deviations from the ideal stoichiometry. Nevertheless, the intensity does not seem to have changed as indicated by the $\mathrm{N}$ signal being approximately constant in the multiplayer range of the 
profile. However, such a shift does not occur in the data presented in Fig. 3d recorded away from the base layer. In this area, the stoichiometry must then be described by $\mathrm{V}_{1-x} \mathrm{Ti}_{y x} \mathrm{Al}_{(1-}$ ${ }_{y) x} \mathrm{~N}$, which means that the maxima of the $\mathrm{Al}$ distribution are presumably displaced against the $\mathrm{V}$ concentration minima opposite to the Ti maxima. This phenomenon may be caused by instabilities during the deposition process. The deposition unit was set to maintain a constant partial pressure of nitrogen via an automatic feedback control. When changing deposition from the $\mathrm{VN}$ base layer to TiAlN/VN multilayer, the metal atom flux from $\mathrm{Ti}$ and $\mathrm{Al}$ increases significantly as soon as the TiAl cathodes start running at full power, hence more nitrogen is required. There is a short time lag between the demand for more $\mathrm{N}_{2}$ and the correct level being achieved in the chamber. Thus, during this period the flux of $\mathrm{Ti}, \mathrm{Al}$, and $\mathrm{V}$ is greater, which may give rise to the proposed variation of the Ti/Al concentration ratio. Furthermore, the HAADF images in Fig. 3 show a slight increase of layer thickness near the base layer which is probably caused by the same mechanism.

There is a curvature in the middle of the VN base layer surface shown in Fig.3a. The curvature of the interface between the base layer and the multiplayer system is probably caused by the initial stage of deposition. V-ion etching was performed using one of the $\mathrm{V}$ targets in cathodic arc mode prior to VN base layer (deposited using magnetron sputtering), which can lead to growth defects in coating, with the number and size depending on the melting point of the target materials [22].

Fig. 4 gives the modelling results derived for TiAlN/VN using the deposition geometry used for the present sample. Fig. 4a shows the time dependent deposition rate of TiAl, V and the total of the system at the substrate, derived as described in Zhou et al. [13].

$$
R(t)=\sum R_{i}(t)=-\sigma \sum_{i} j_{i} \frac{m_{i}}{\rho_{i}} \cos \alpha=\frac{\mathrm{d} d}{\mathrm{~d} t} \quad \text { (Equation 1) }
$$

$i$ denoting the different evaporated species. The cross section $\sigma$ is added here and is accounting for the part of particles arriving at the substrate which really are incorporated in the layer (sticking coefficient). It is not known, thus, the time has to be given in arbitrary units. However, it is assumed to be equal for both V and TiAl.

It follows straightforwardly that the layer thickness over time is obtained by integrating $R$ :

$d(t)=\int R(t) \mathrm{d} t \quad$ (Equation 2),

We may assume the sum of the relative concentrations being constant: 
$C_{\mathrm{V}}+C_{\mathrm{TiAl}}=$ const $\quad($ Equation 3$)$,

and therefore we can write the concentrations of either of the two species as

$$
C_{i}(d)=\frac{R_{i}(d(t))}{R_{\mathrm{V}}(d(t))+R_{\mathrm{TiAl}}(d(t))} \quad(\text { Equation } 4) .
$$

To circumvent the analytical problem of inverting the function $d(t)$ for the parameterisation, we used a numerical representation of $d(t)$ which was derived from the modelled $R(t)$ anyway. It is straightforward to swap the argument for the $R$ data from $t$ to $d$, however, the numerical $R$ data values are not equidistant in $d$ which is necessary for further numerical treatment. Therefore, the data were interpolated to a grid of 16384 equidistant points.

To account for interface mixing, the thus obtained raw profiles were convoluted with a function of the form $\exp (-|d / \delta|)$. This form should account for diffusion processes, but comes also close to the intensity profile of the STEM probe used in the investigation. Further influences such as instabilities of the probe position with respect to the sample could probably be regarded by a further convolution with a Gaussian, but this was not done. Fig. 4b shows obtained concentration profiles for $\mathrm{V}$ for different parameters $\delta$ as well as the raw profile before applying the convolution. A detailed investigation into the type of broadening functions would require a much lower noise level of the experimental data than available and also a much better defined sample geometry, especially completely plain layers with an exactly determinable orientation. This condition, however, is not fulfilled.

Experimental concentration profiles are shown in Fig. 5. Since the absolute intensities of the EELS signals might be influenced by the geometry and orientation of the crystalline sample and are influenced by instabilities in the emission current of the microscope's electron gun, we can only give relative concentrations. Again, we assume a Ti/Al concentration ratio $C_{\mathrm{Ti}} / C_{\mathrm{Al}}$ of 1 . Then, using Equation 3, the relative concentrations of $\mathrm{VN}$ and TiAlN follow straightforwardly:

$$
C_{\mathrm{VN}}=\frac{C_{\mathrm{V}}}{C_{\mathrm{V}}+2 C_{\mathrm{Ti}}}, \quad C_{\mathrm{TiAlN}}=\frac{2 C_{\mathrm{Ti}}}{C_{\mathrm{V}}+2 C_{\mathrm{Ti}}} \text { (Equation 5). }
$$

The concentrations $C_{\mathrm{V}}$ and $C_{\mathrm{Ti}}$ are determined from the measured net EELS intensities (Fig.3c and d) by weighting with inelastic cross section, as shown in Fig.3e and f. The depth scale of the simulated profile was calibrated from the average periodicity of the experimental data. This again was derived from HAADF images taken immediately before and after the EEL data were recorded to identify and correct for the drift during the EELS 
measurement. The scale of the HAADF images is known to within $1 \%$. Due to the setting of the rotation frequencies of the substrate during deposition, the profile fine structure repeats mainly every third period (see also Fig. 4b). This seems to be in contradiction with the stronger variation of the amplitude of the time dependent deposition rate as shown in Fig.4a, but the varying peak heights are counterbalanced by the contrarily varying peak widths. The amplitude of the measured concentration is not constant. However, the experimental data extend over too less slabs to identify a periodicity, the only comparable ranges are $d=(0.0 \ldots 4.7) \mathrm{nm}$ and $d=(10.5 \ldots 15.2) \mathrm{nm}$ which do not resemble each other to a large extent, neither regarding the overall values nor the fine structure.

The model predicts that there will be interfacial mixing over a region of $\sim 1 \mathrm{~nm}$, but that each layer will contain components of the adjacent layer, i.e. that $\mathrm{Ti}$ and $\mathrm{V}$ will be present throughout the sample.

The model was derived from a geometric analysis of the flux, and does not take account the crystallography of the interface. Thus, the model prediction of the compositional modulation within an individual layer is determined by the geometry of deposition, and does not take account of the inevitable differences between a $\{111\},\{220\}$ and $\{200\}$ interface. In the current experimental work, the region examined exhibited (220) parallel to the layers which would suggest that the compositional modulation within each layer should occur across approximately ten (220) planes (with $d_{220}=0.15 \mathrm{~nm}$ and $d_{\mathrm{VN}}=\sim 1.5 \mathrm{~nm}$ ). The EELS intensity profiles do not reveal fine structure in composition within the individual layers. The steepest slopes of the experimental profiles in Fig. $4 \mathrm{c}$ do coincide with those of the simulated profiles after convoluting with the wider diffusion profile. That implies that the parameter $\delta$ has a value close to $0.511 \mathrm{~nm}$. The larger blurring of other parts of the experimental profiles may be caused by mainly three sources. Firstly, after the initial tuning of the $\mathrm{C}_{\mathrm{s}}$ corrector, subsequent drift would lead to an increase in probe size. Nevertheless, since atomic resolution was obtained (Fig.2b), probe size increases do not appear to have been sufficient to obscure the predicted compositional modulation. Neither does the sample drift, which was found minimal. The second possible explanation is that curvature in the VN and TiAlN layers occurred through the thickness of the sample. This is obvious for instance in Fig.3b, where some layers deviate from planes by almost $1 \mathrm{~nm}$ over the shown image range. Interestingly, the atomic resolution obtained in conventional HREM images of regions with a similar sample thickness suggests that the extent of curvature was not sufficient to obscure the 
compositional modulation. Thirdly, the coating was found highly faulted with a complex sub-grain structure [23], which could also obscure the compositional modulation.

Nevertheless, there is excellent agreement between the width of the interface predicted by the model and the experimental data, which suggests an interface region of $\sim 1 \mathrm{~nm}$. Moreover, the experimental observation of $\mathrm{V}$ and $\mathrm{Ti}$ throughout the coating, such that no individual layer is pure $\mathrm{VN}$ or TiAlN, is again consistent with the model. The intermixing of layers will have reduced the interfacial coherency strains and Koehler force arising from modulus mismatch. A more subtle, but clearly defined, prediction of the model is that the thickness of the individual layers varies with coating thickness, exhibiting systematic variations. This is the result of 3-fold rotation used during deposition. Fig. 5 shows the spacing of subsequent $\mathrm{V}$ layers of the data from Fig. 4. In order to estimate the centre of gravity of the individual V layers, we convoluted the data with a rather broad Gaussian peak of 1.0nm FWHM and determined the positions of the maxima in the resulting smooth curve. Apart from an overall decrease in the spacing from approximately $3.4 \mathrm{~nm}$ to $2.9 \mathrm{~nm}$, one recognizes a modulation with a frequency slightly higher than $1 / 3$, approximately $(1 / 3+1 / 27)$, in terms of layer numbers (and thus rotations of the turntable during deposition) Layer thickness measurements by EFTEM images and Fresnel HAADF Z-contrast images by Zhou et al. [13] confirmed the presence of this modulation. The current EELS line scans are consistent with the model prediction. We find a distinct variation of the layer thickness in the profile shown in Figs. 3f and 4c. We matched the variation of layer thicknesses in the experimental and simulated profiles with $f=1 / 3$ by adjusting an offset of the simulated data but albeit was not possible to determine the phase shift between the first and second rotation mainly because the total length of the line-scan used was too small to fully investigate this aspect of the coating structure. In any event, this modulation is unlikely to have a strong effect on mechanical properties, but would be expected to affect the coating's optical properties.

\section{Summary}

TiAlN/VN multilayer films with an average layer thickness, $\lambda=3.4 \mathrm{~nm}$ were produced by unbalanced magnetron sputtering in a commercial scale deposition unit, that employs 3 axis rotation of the specimen to ensure uniform deposition on complex shapes. The crystallographic texture of the films varied throughout the thickness, with (200) preferentially 
parallel to the coating plane near the substrate and (111) preferentially parallel near the outer regions. Spherical aberration (Cs) corrected STEM allowed chemical specific high angle annular dark field (HAADF) images to be generated that revealed the atomic structure of the interface between layers. The interface was found to be rough, with local thickness variations common. Electron energy loss spectroscopy (EELS), using a $0.1 \mathrm{~nm}$ probe (which had revealed the atomic structure), demonstrated significant chemical mixing between layers. The interface between layers was around $1 \mathrm{~nm}$ thick and the VN contained small amounts of $\mathrm{Ti}$, while the TiAlN contained small amounts of $\mathrm{V}$. The experimental results are fully consistent with previously published numerical modelling of the deposition fluxes for this system. Evidence for unbalanced concentration ratio of $\mathrm{Ti}$ and $\mathrm{Al}$ was found in the region of the multiplayer system adjacent to the base layer.

\section{Acknowledgement:}

Financial support from Engineering and Physical Science Research Council (EPSRC), UK, Grant No. GR/N23998/01 is acknowledged.

\section{References}

[1] Scripta Materialia View Point Set No.34: Deformation and Stability of Nanoscale Metallic Multilayers, Scripta Mater., 50, 707 (2004).

[2] S.A. Barnett, and A Madan, Scripta Mater., 50, 739 (2004).

[3] X. Chu, and S.A. Barnett, J. Appl. Phys., 77, 4403 (1995).

[4] P.M. Anderson, and C. Li, Nanostructured Mater., 5, 349 (1995).

[5] R.G. Hoagland, R.J.Kurtz, and C.H. Henager Jr, Scripta Mater., 50,775 (2004).

[6] W.-D. Münz, D.B. Lewis, P.Eh. Hovsepian, C. Schonjahn, A. Ehiasarian, and I.J. Smith, Surf. Eng., 17, 15 (2001).

[7] L.H. Friedman, Scripta Mater., 50,763 (2004).

[8] F. Spaepen, and D.Y.W. Yu, Scripta Mater., 50, 729 (2004).

[9] B. Rother, H.A. Jehn, and H.M. Gabriel, Surf. \& Coat. Tech., 86-87, 207 (1993).

[10] B.Rother, and H.A. Jehn, Surf. \& Coat. Tech., 62, 635 (1993).

[11] B.Rother, Surf. \& Coat. Tech., 64,155 (1994).

[12] B.Rother, G.Ebersbach, and H.M.Gabriel, Surf. \& Coat. Tech., 116-119, 694 (1999).

[13] Z. Zhou, W.M. Rainforth, B. Rother, A. Ehiasarian, P.Eh. Hovsepian, and W.-D. Münz, Surf. \& Coat. Tech., 183,275 (2004).

[14] C. Colliex, and C. Mory, in quantitative electron microscopy, edited by J.P Chapman and A.J. Craven, SUSSP publications, Edinburg, 1984, pp149-216.

[15] R.F. Egerton, in Electron energy loss spectroscopy in the microscope, $2^{\text {nd }}$ edition, kluwer Academic/plenum publishers, 1996, pp345-346.

[16] D.A. Muller, and J. Silcox, Ultramicrsocopy, 95, 195 (1995).

[17] D.B. Lewis, L.A. Donohue, M. Lembke, W.-D. Münz, R. Kuzel, V. Valvoda, C.J. Blomfield, Surf. \&Coat. Tech., 114, 187 (1999). 
Fig. 2. (a) Phase contrast HREM image (JEOL2010F) from the multilayer close to the outer edge of the coating. The image fails to reveal the position of the individual layers, but confirms coherency (layers running approximately left to right). (b) HAADF image from a similar region to (a), taken on the $\mathrm{C}_{\mathrm{s}}$ corrected STEM in Daresbury, showing similar atomic information to (a), but with the position of the layers clearly identified through the $\mathrm{Z}$ contrast.

Fig. 3. HAADF image from (a) adjacent to the base layer, i.e. a similar region to Fig. 1 and (b) from the same region as Fig. 2, towards the outer region of the coating. (c,d) Net intensities of the N-K, Ti-L and V-L loss edges from regions marked in $(a, b) .(e, f)$ relative concentrations of VN and TiAlN by equation 5. Solid: V-L, dotted: Ti-L, dashed: N-K.

Fig. 4 (a) Numerical calculation of the time dependent deposition rate of $\mathrm{V}$ and $\mathrm{Ti}$, and of the total system. (b) Concentration profiles for V. Grey: raw simulated profile, Black: raw profile convoluted with different mixing profiles $(\delta=(0.204,0.255,0.341,0.511) \mathrm{nm})$. (c) Profiles of relative concentrations for VN and TiAlN. Solid: experimental results as Fig.3f. Dotted: Simulated profiles for $\delta=0.341 \mathrm{~nm}$ and $\delta=0.511 \mathrm{~nm}$. The simulated profiles are shifted with respect to (b) to find good agreement between experimental and simulated profiles with respect to the varying layer thickness.

Fig.5 Interlayer spacing of V layers in the simulated data in Fig.4. 
Table 1 Energy windows for background subtraction and edge integration

\begin{tabular}{|l|l|l|l|}
\hline & $\mathrm{N}-\mathrm{K}(401 \mathrm{eV})$ & $\mathrm{Ti}-\mathrm{L}_{2,3}(456 \mathrm{eV})$ & $\mathrm{V}_{-} \mathrm{L}_{2,3}(513 \mathrm{eV})$ \\
\hline Range at pre-edge for & $370-400$ & $420-456$ & $475-509$ \\
\hline $\begin{array}{l}\text { Range at edge for edge } \\
\text { integration }\end{array}$ & $406-450$ & $460-506$ & $515-570$ \\
\hline
\end{tabular}


Fig.1

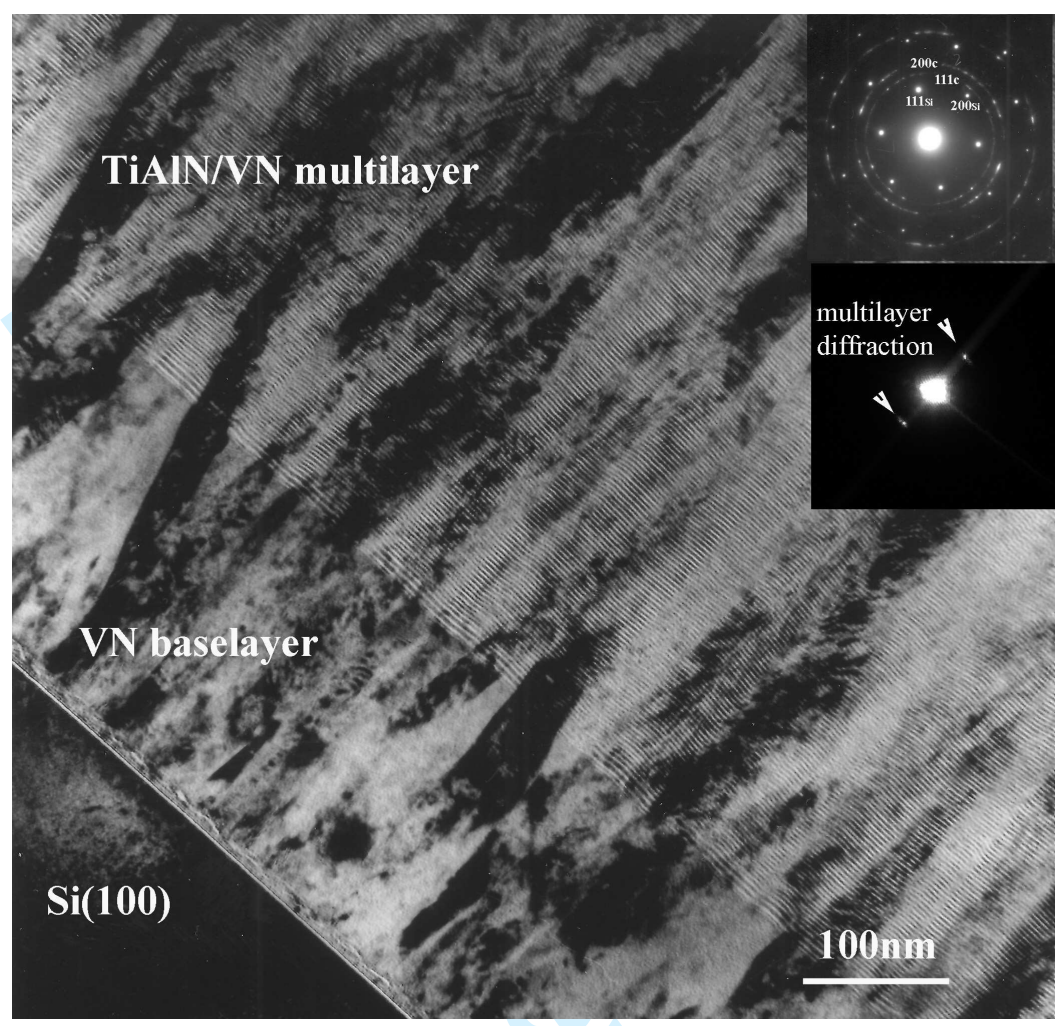


Fig.2

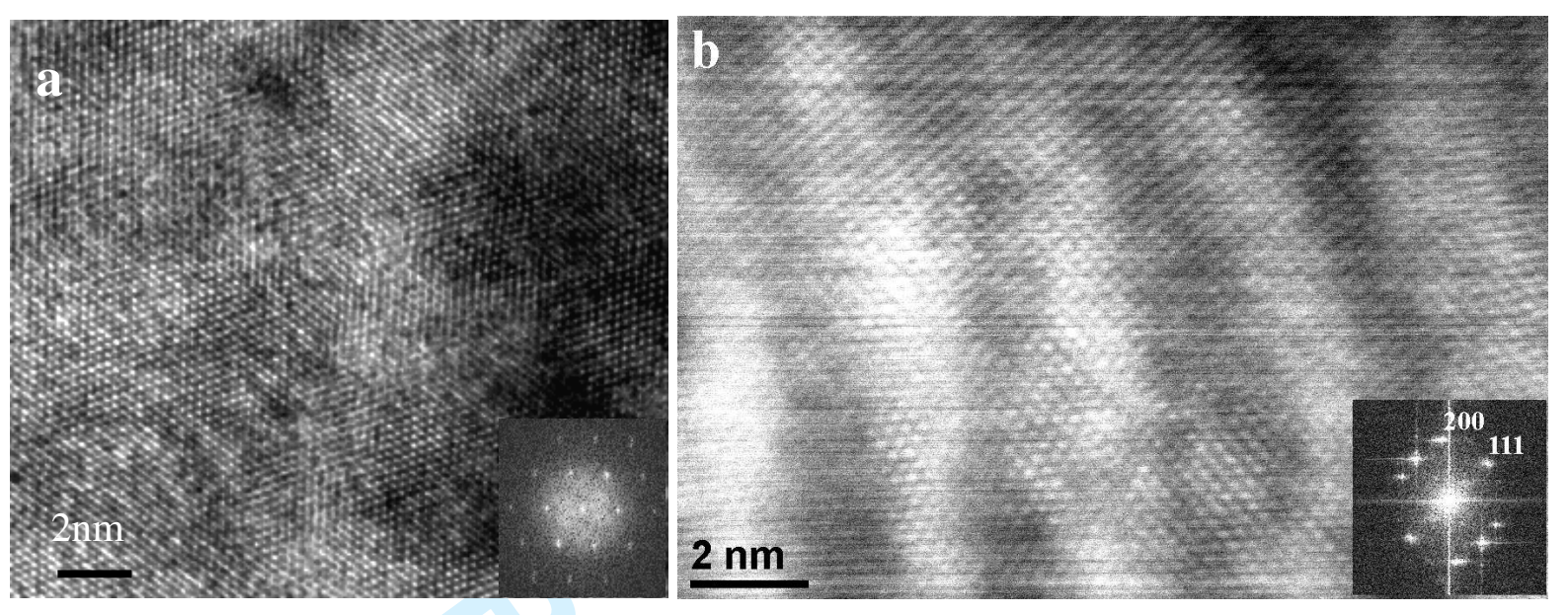


Fig.3
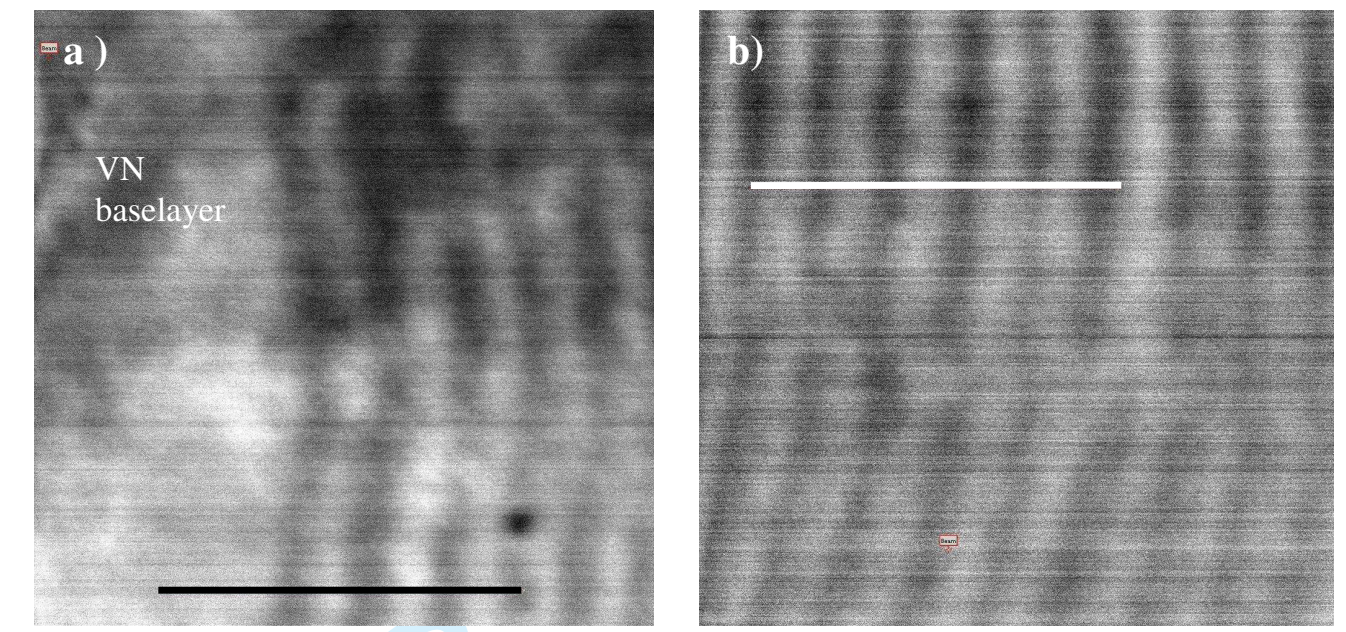

a

b
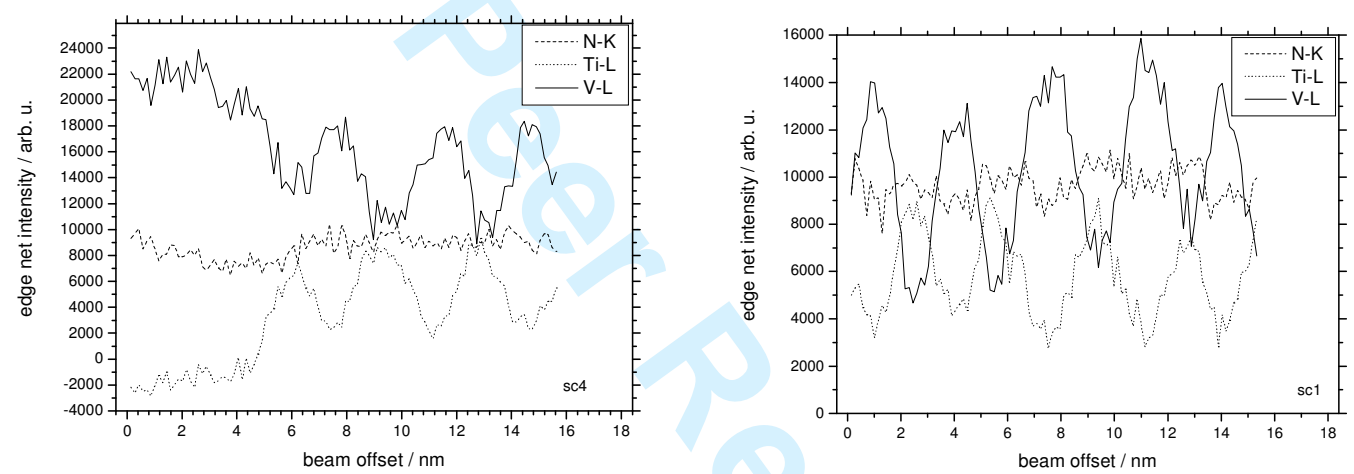

c

d
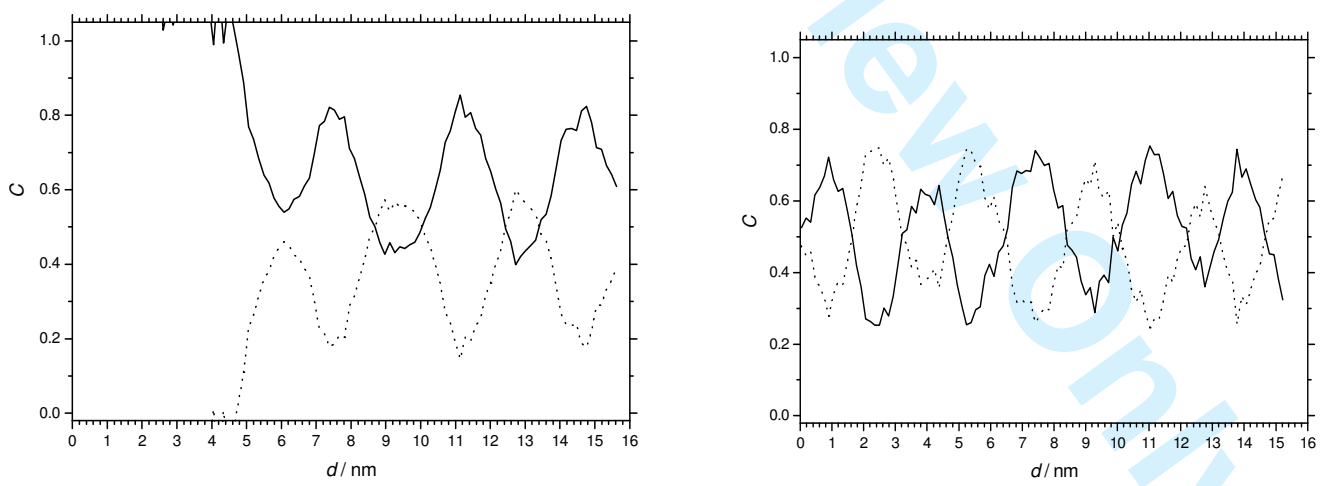

e

f 
Fig.4

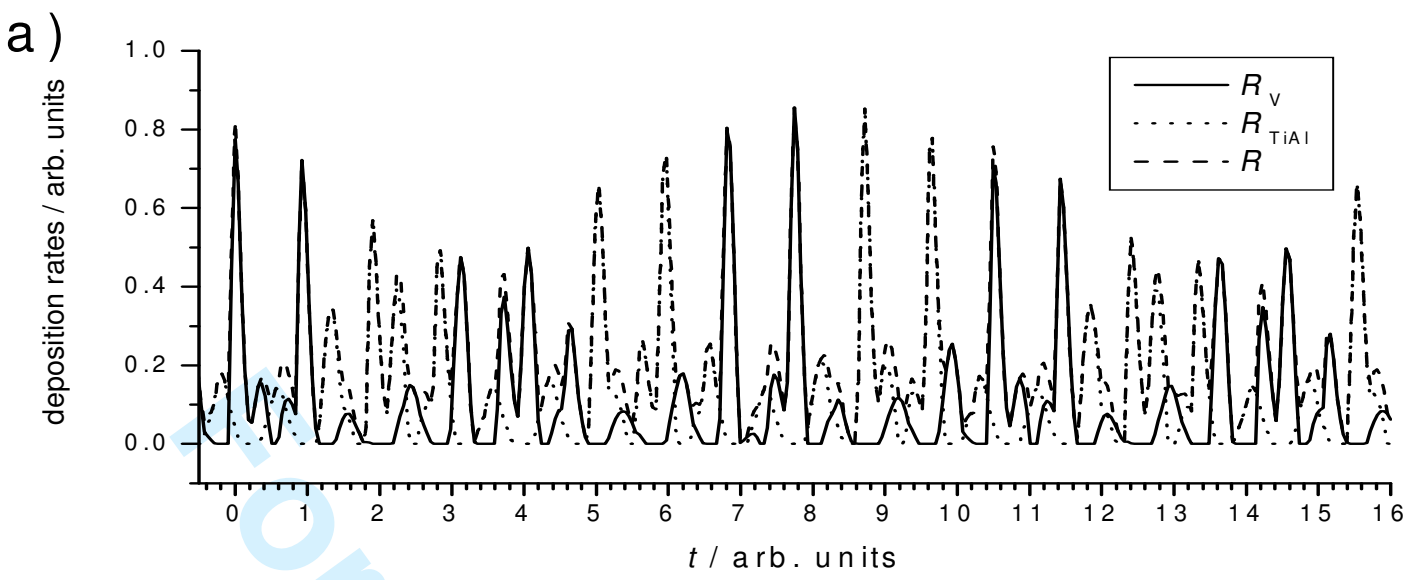

b)
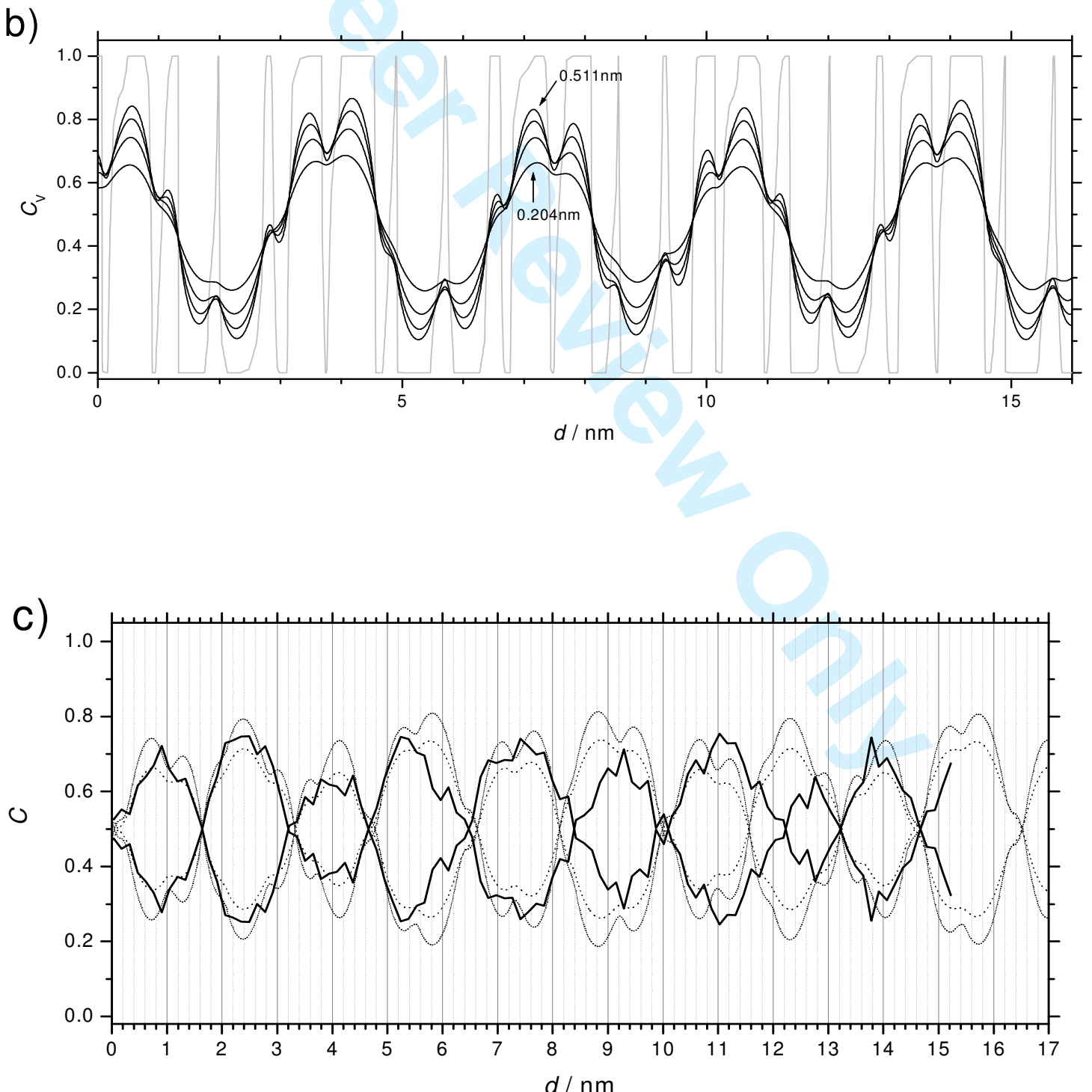
Fig. 5

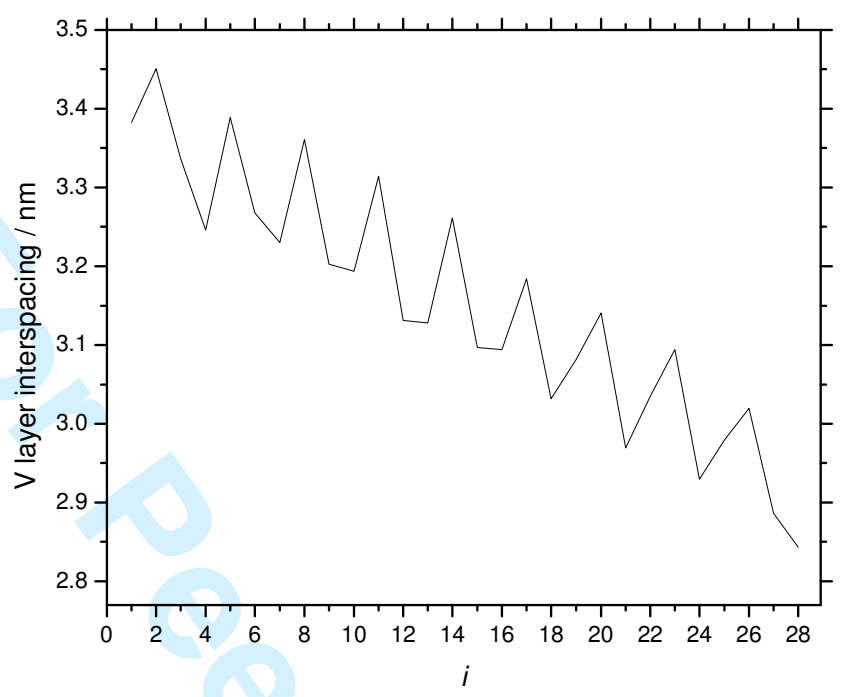

28

29

30

31

32 